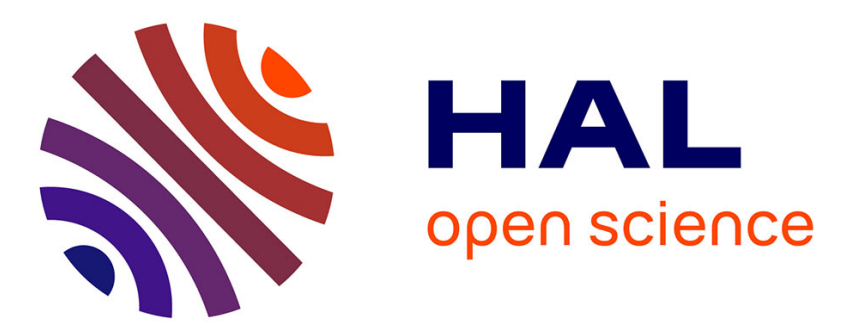

\title{
L'identité professionnelle des enseignants d'EPS : entre valeurs partagées et interprétations singulières
}

Thérèse Roux-Perez

\section{To cite this version:}

Thérèse Roux-Perez. L'identité professionnelle des enseignants d'EPS : entre valeurs partagées et interprétations singulières. STAPS : Revue internationale des sciences du sport et de l'éducation physique, 2004, 63 (1), pp.75-88. 10.3917/sta.063.0075 . hal-01716418

\section{HAL Id: hal-01716418 \\ https://hal.science/hal-01716418}

Submitted on 23 Feb 2018

HAL is a multi-disciplinary open access archive for the deposit and dissemination of scientific research documents, whether they are published or not. The documents may come from teaching and research institutions in France or abroad, or from public or private research centers.
L'archive ouverte pluridisciplinaire HAL, est destinée au dépôt et à la diffusion de documents scientifiques de niveau recherche, publiés ou non, émanant des établissements d'enseignement et de recherche français ou étrangers, des laboratoires publics ou privés. 


\section{Titre :}

L'identité professionnelle des enseignants d'EPS: entre valeurs partagées et interprétations singulières

\section{Résumé}

L'article se donne pour objet d'investir les éléments constitutifs d'une identité collective au sein du groupe professionnel des enseignants d'Education Physique et Sportive (EPS). Une enquête par questionnaire permet d'appréhender la profession à partir des valeurs et des représentations professionnelles partagées. L'utilisation complémentaire d'entretiens semidirectifs nuance cette approche et rend compte des distorsions entre un discours collectif relativement consensuel et des interprétations singulières. Un certain nombre de tensions apparaissent alors entre spécificité et intégration de la discipline au sein du système scolaire, entre logique sportive et logique d'apprentissage de l'élève, entre pratique et théorie. Pour dépasser les clivages et retrouver une unité, les enseignants d'EPS se réfèrent aux finalités de la discipline, aux valeurs éducatives et revendiquent une relation privilégiée avec les élèves, se situant alors comme des acteurs particuliers et indispensables au sein de l'école.

Mots clés : identité professionnelle. Représentations. Valeurs. EPS

\section{Title}

The professional identity of physical education teachers : from shared values to singular interpretations.

\section{Summary}

The article aims to invest the constitutive elements of a collective identity among the professional group of physical education teachers. An investigation carried out through questionnaires permits to apprehend the profession from the shared professional values and representations. The complementary use of semi-directive interviews qualifies this approach and gives an account of the distortions between a quasi-consensual collective discourse and singular interpretations. A certain amount of tensions appears, hence, between the specificity of the discipline and its integration amidst the academic system, between the sports'logic and the pupil's learning logic, between the practice and the theory. In order to overcome the cleavages and get unity back, the sports'teachers refer to the finalities of the discipline and to educational values. They claim a privileged relationship with the pupils as they set themselves as essential and special actors within schools.

\section{Key} words

Professional identity. Representations. Values, Sportive and Physical Education. 


\section{L'identité professionnelle des enseignants d'EPS : entre valeurs partagées et interprétations singulières}

\section{Introduction}

\subsection{Le contexte institutionnel}

Le fait que l'Education Physique et Sportive (EPS) n'ait une spécificité que "dans, par et pour l'école" (Arnaud, 1989, 29), amène les enseignants d'EPS à construire leur identité professionnelle dans une relation particulière avec cette discipline scolaire. Or, durant les 30 dernières années, l'EPS a vécu de profondes mutations institutionnelles officialisées par la parution de nombreux textes organisant la discipline. Une étude préalable de ces textes durant la période 1967-2000 (Roux-Perez, 2001), a permis d'identifier un ensemble d'éléments révélateurs de l'évolution disciplinaire. Une analyse de contenus, réalisée à partir de certains « objets professionnels » (Moliner, 1993) ${ }^{1}$, a mis en relief continuités et changements au sein de la discipline : continuité des finalités, des valeurs et des objectifs fondant en quelque sorte une identité disciplinaire ; changement dans le statut accordé à l'élève qui devient acteur de ses apprentissages. Placer l'élève au centre des préoccupations éducatives a induit la transformation des pratiques professionnelles : construction de projets pédagogiques, traitement didactique des Activités Physiques Sportives et Artistiques (APSA), réflexion sur les contenus à enseigner et sur les modalités d'évaluation, mise en place de procédures pédagogiques nouvelles (différenciation, évaluation formative, pédagogie du contrat, etc.).

Cette évolution souhaitée par l'institution et repérable à travers les textes a été appréhendée de façon très différente par les enseignants d'EPS qui, pour certains, se sont inscrits dans la dynamique de changement alors que d'autres, se sentant en décalage avec les compétences professionnelles nouvelles exigées par l'institution ont eu tendance à résister à ce changement en estimant que l'EPS était en train de «perdre son identité ».

En fait, la question de l'identité disciplinaire revient régulièrement dans les débats qui traversent la profession. Souvent controversée, elle semble pourtant conserver une valeur mythique (Prévost, 1991) et fonder, au moins en partie, l'identité professionnelle des enseignants d'EPS envisagée dans sa dimension collective.

\subsection{Une identité professionnelle entre unification et distinction}

L'identité professionnelle inclut l'identification à une discipline dans laquelle l'enseignant se reconnaît plus ou moins (Berger, 1989). En effet, l'ancienne vision culturaliste selon laquelle des éléments rituels ou symboliques assuraient l'intégration des membres de la société s'est complexifiée ; on pense plutôt aujourd'hui que les individus entretiennent des liens multiples et surtout changeants avec leurs communautés et leurs croyances. Par ailleurs, les formes d'identification par les institutions (instructions, programmes) constituent une voie intéressante pour défendre la profession et lui donner une identité. Ainsi, l'identité professionnelle, "c'est ce qui permet aux membres d'une même profession de se reconnaître eux-mêmes et de faire reconnaître leur spécificité à l'extérieur. Elle suppose donc un double travail d'unification interne d'une part, de reconnaissance externe d'autre part" (Ion, 1990, 91). Modèles professionnels, institutions de formation, culture de métier, communauté de pratiques, organisations syndicales, participent à la construction des identités professionnelles. De ce point de vue, Couturier et Duret (2000) ont questionné l'identité professionnelle des enseignants d'EPS dans sa relation avec le sport en étudiant les rapports entre leur degré

\footnotetext{
${ }^{1}$ Les "objets professionnels" retenus dans l'analyse de contenu concernent les finalités et objectifs de la discipline, les programmes, le projet pédagogique, les contenus d'enseignement, l'évaluation, le statut de l'élève, l'apprentissage, le traitement des Activités Physiques Sportives et Artistiques (APSA), les modalités de groupement des élèves.
} 
d'investissement par rapport à la pratique sportive et leur conception de l'Education Physique. Souhaitant donner un éclairage sur les représentations de la fonction enseignante en lien avec les évolutions qui traversent et réorganisent ce corps professionnel, les auteurs « repèrent et quantifient opinions et attitudes, facilitant ainsi la compréhension des raisons mobilisatrices, qui, par delà leurs disparités, permettent aux différents segments d'enseignants de se reconnaître dans la profession" (Couturier et Duret, 2000, 2). De plus, ils soulignent la recherche d'intercompréhension entre des enseignants qui, par delà leurs oppositions, ont en commun le souci de défendre des valeurs. Ces valeurs sont l'occasion de prendre position au sein de l'école et d'exister face aux représentations véhiculées par les différents acteurs sur la discipline EPS et sur les enseignants qui la mettent en œuvre dans leurs pratiques quotidiennes.

En effet, le processus d'unification au sein de la profession ne peut être pris en compte sans analyser les phénomènes de reconnaissance externe. Croiser les regards portés sur l'identité des enseignants d'EPS donne un aperçu de cette "identité pour autrui" (Mead, 1934; Sainsaulieu, 1977; Tap, 1980) constitutive de l'identité professionnelle. De nombreux travaux ont porté sur les représentations de l'EPS et de ses enseignants pour les parents (Dorvillé, 1991 ; Gleyse, 1993) pour les élèves (Hebrard, 1986 ; Josse, 1975 ; Beunard, 1999) ou pour les enseignants des autres disciplines (Dorville, 1991 ; Gleyse, 1993). Les résultats éclairent la manière dont les différents acteurs du système scolaire définissent l'enseignant d'EPS à travers des qualités relationnelles et d'animation, dans une discipline organisée autour d'une dimension essentiellement éducative.

En fait, la spécificité d'un travail sur le corps donne à celui-ci le privilège d'une proximité avec les élèves mais le distancie des valeurs intellectuelles de l'école. Ainsi, parler du corps et des pratiques sportives, c'est être reconnu spécifiquement mais de façon marginale ; mettre le corps à distance et s'engager dans une perspective plus générale de formation, c'est risquer d'entrer dans une "orthodoxie scolaire" (Arnaud, 1983) et remettre en cause les points d'unification au sein de la profession (Gleyse, 1993).

Ainsi, la socialisation professionnelle s'inscrit dans un double processus d'unification et de distinction, fondateur de l'identité disciplinaire. En effet, il existe une tension permanente entre l'intégration de l'EPS comme discipline d'enseignement au sein du système éducatif et la part de différenciation / distinction revendiquée à travers l'affirmation d'une spécificité disciplinaire.

Cette recherche d'intégration et de différenciation se retrouve à l'intérieur du groupe professionnel : le sentiment d'appartenance à une communauté n'empêche pas le sujet de se penser comme unique et différent des autres. En ce sens, la notion d'identité est paradoxale : elle désigne ce qui est unique, ce qui distingue et différencie des autres, mais elle qualifie aussi ce qui est identique, c'est-à-dire semblable, tout en étant distinct. Cette ambiguïté sémantique a un sens profond : elle suggère que l'identité oscille entre similitude et différence, entre ce qui fait de l'individu une identité singulière et qui, dans le même temps le rend semblable aux autres (Tap, 1998). Ce paradoxe se retrouve aussi au niveau de l'identité disciplinaire.

Prenant appui sur ces différents travaux de recherche, l'étude réalisée entre 1998 et 2001 s'est donnée pour objet d'appréhender la question de l'identité professionnelle à l'échelle du collectif des enseignants d'EPS en étudiant les représentations de ce groupe sur un certain nombre d'objets professionnels qui organisent la discipline.

\subsection{Des représentations partagées constitutives d'une identité professionnelle}

Les enseignants d'EPS véhiculent un ensemble de valeurs et de croyances, partagées avec certains autres enseignants, compatibles avec leurs pratiques professionnelles ou personnelles et repérables à travers leurs représentations du métier, des autres et d'eux-mêmes (Couturier et 
Duret, 2000 ; Roux-Perez, 2001). En construisant ces représentations, ils réduisent d'éventuelles dissonances avec leur environnement humain (les autres acteurs) et institutionnel (les textes officiels). Les théories des représentations sociales (Abric, 1976 ; Jodelet, 1984 ; Moscovici,1961) et professionnelles (Blin, 1997) éclairent ce point de vue ; elles permettent d'étudier la manière dont les enseignants perçoivent l'évolution de leur discipline et justifient leurs positions vis-à-vis du métier. Formes de connaissances porteuses de valeurs, les représentations sociales donnent sens à la pratique et légitiment une certaine "vision du monde" (Moscovici, 1961). Si la représentation rend compte des relations complexes, réelles ou imaginaires qu'un sujet entretient avec un objet (Abric, 1994), elle intègre aussi des interactions sociales et devient une "forme de connaissance socialement élaborée et partagée ayant une vision pratique et concourant à la construction d'une réalité commune à un ensemble social" (Jodelet, 1989, 36).

Par ailleurs, les représentations sont structurées autour d'un noyau central (Abric, 1976 ; Flament, 1987) auquel sont associés des éléments périphériques. Si le noyau central, très résistant au changement, offre un caractère de cohérence et de stabilité à la représentation, le système périphérique, plus souple et plus sensible aux effets de contexte, permet l'intégration dans la représentation des variations individuelles liées à l'histoire des sujets et déterminées par des expériences spécifiques. De ce point de vue, les représentations sont à la fois stables et mouvantes, rigides et souples ; elles servent à agir et réagir face à l'environnement tout en conservant un équilibre cognitif dans un contexte professionnel particulier.

Ainsi, les représentations professionnelles, considérées comme l'ensemble des idées que partagent les individus sur un métier donné, sont d'abord fonctionnelles (Blin, 1997). Elles permettent aux acteurs de comprendre et d'agir sur la réalité selon un principe d'économie cognitive favorable à la résolution de problèmes quotidiens au moindre coût. Elles définissent les identités professionnelles et protègent la spécificité des groupes intra et inter professionnels. De plus, les représentations professionnelles sont spécifiques : elles sont construites par des acteurs dans les actions et interactions professionnelles qui les contextualisent. En ce sens, elles prennent appui sur des "objets "significatifs dans le champ professionnel considéré.

Enfin, les représentations professionnelles sont sous-tendues par des valeurs qui

mobilisent le sujet et l'engagent dans l'action (Mias, 1998). De ce point de vue, elles constituent des matrices d'action contribuant à la construction des identités professionnelles (Barbier, 1996 ; Camilleri, 1990 ; Dubar, 1991 ; Barbier et Galatanu, 1998 ; Tap, 1980).

\section{Méthode}

Une enquête par questionnaire auto-administré a été réalisée en 1998 dans trois académies aux conditions d'enseignement différentes : Nantes, Créteil et la Corse. Les 381 retours sur les 654 questionnaires envoyés $^{2}$ ont offert des garanties de représentativité par rapport à la population nationale du point de vue du sexe, de l'âge, du type d'établissement d'affectation et du statut des enseignants au moment de l'enquête. Au-delà des questions classiques d'identification, le questionnaire interrogeait l'histoire familiale et sportive, la formation initiale et continue, le parcours professionnel et le rapport au métier. Ce dernier point était envisagé à travers : a) la relation à l'équipe d'éducation physique de l'établissement; b) les difficultés rencontrées dans le contexte professionnel; c) les différentes tâches remplies par l'enseignant à l'intérieur ou à l'extérieur du système scolaire.

Par ailleurs, une série de questions ouvertes avait pour but de comprendre comment les enseignants d'EPS analysaient l'évolution de la discipline et de le référer : a) au degré de

\footnotetext{
${ }^{2} 654$ questionnaires ont été envoyés pour diffusion auprès des enseignants d'EPS : 100 dans l'académie de Corse, 200 dans l'académie de Créteil et 354 dans l'académie de Nantes (soit un par établissement).
} 
motivation exprimé pour l'enseignement; b) à la perception des textes officiels ; c) aux changements observés dans les pratiques professionnelles depuis l'entrée dans le métier. Une analyse de contenus a été réalisée autour des objets professionnels révélés par les réponses.

A cela s'ajoutaient des questions à choix multiple ordonné permettant de repérer les représentations du métier: la première invitait l'enseignant à choisir trois activités professionnelles dont il se sentait le plus proche dans l'exercice de sa profession (éducateur, acteur-comédien, bricoleur, animateur, entraîneur, concepteur, technicien, accompagnateurguide, militant, autre); la seconde portait sur l'idéal professionnel abordé à travers le choix de trois items dans un référentiel de quinze compétences issu des travaux de Paquay et Wagner $(1996)^{3}$;

Les données ont été traitées avec le logiciel d'enquête Modalisa (version 3.8). Une partie des résultats, issue essentiellement des tris à plat, a permis d'appréhender ce qui pourrait fonder une identité professionnelle collective. A la suite de cette opération, des tris croisés (profil de modalités) ont été systématisés pour prendre en compte les modalités en attirance (Khi 2 au seuil significatif de 0.05) ; l'intitulé de ces modalités a été retranscrit entre guillemets et en italique dans le texte.

Une enquête qualitative a complété ces premières données. Des entretiens semi-directifs ont été effectués avec vingt et un sujets durant l'année 1999-2000, sur la base d'une typologie fondée sur les modes d'implication privilégiés au sein ou à l'extérieur du système scolaire ${ }^{4}$. Douze d'entre-eux ont été choisis et traités à partir d'une analyse structurale du discours (Demazière et Dubar, 1997) en repérant : a) les séquences ou épisodes du récit qui jalonnent le parcours ; b) les actants ou personnages qui interviennent dans le récit et avec lesquels s'établissent des réseaux de relations; c) les propositions ou arguments (représentations) destinés à convaincre l'interlocuteur, à défendre son point de vue (valeurs, identité pour soi et pour autrui, rapport à l'institution). Par ailleurs, l'intérêt de cette démarche consistait à revisiter certaines réponses du questionnaire pour en approfondir le sens donné par les sujets interrogés ${ }^{5}$. Cette approche complémentaire a permis de mettre à jour les cohérences et les distorsions entre un discours collectif relativement consensuel et des interprétations singulières.

\section{Résultats}

\subsection{La connaissance du groupe professionnel}

Des résultats différenciés apparaissent en fonction du sexe, de l'âge, de la pratique sportive, de la localisation géographique, du degré de motivation pour le métier et de difficultés rencontrées dans l'exercice de la profession.

\footnotetext{
${ }^{3}$ La question était formulée comme suit : "un enseignant d'EPS c'est, avant tout, une personne qui : a) maîtrise bien les APSA ; b) sait construire des contenus pour que l'élève progresse ; c) connaît bien la psychologie de l'adolescent ; d) sait utiliser les savoir-faire techniques ; e) sait appliquer les règles organisant les pratiques physiques ; f) sait situer où sont les erreurs par rapport au geste le plus efficace; g) sait s'engager dans des projets collectifs ; h) développe des enjeux sociaux dans les situations quotidiennes ; i) sait être en relation ; $j$ ) cherche à évoluer ; $k$ ) recherche un développement personnel ; l) réfléchit sur ses pratiques et analyse leurs effets $; m)$ cherche à produire des outils innovants; $n$ ) réalise les différentes tâches qui lui sont assignées;o) sait s'adapter rapidement au contexte du cours ; p) autre".

${ }^{4}$ Cette étude est développée dans un article intitulé "Identité professionnelle et modes d'implication privilégiés chez les enseignants d'EPS" (2003, Revue Les Sciences de l'Education pour l'Ere Nouvelle.

${ }^{5}$ Un prénom a été attribué aux enseignants interviewés, issus de la typologie : Max et Brigitte appartiennent au groupe des "enseignants-entraîneurs", François et Samuel à celui des "compétiteurs", Marie et Claude à celui des "éducateurs", Paul et André à celui des "acteurs institutionnels", Pierre et Mathieu à celui des "critiques" ; Colette et Michèle ont la particularité d'être des enseignantes multi-impliquées, appartenant à plusieurs types.
} 
Tout d'abord, les résultats de l'enquête soulignent des caractéristiques sensiblement différentes selon le sexe des enseignants. En effet, le profil de modalités réalisé à partir de la variable sexe montre que les hommes sont davantage orientés vers des tâches d'entraîneurs et pratiquent encore en compétition. S'ils se forment moins que les femmes au sein de l'éducation nationale, ils optent en revanche pour des stages fédéraux leur permettant l'accès à des diplômes sportifs. Pour eux, l'enseignant "idéal" "maîtrise les APSA" et l'enseignant d'EPS a aussi un rôle de "technicien". De leur côté, les femmes n'ont pour la plupart aucun diplôme sportif et n'ont jamais entraîné en club. "La réussite en EPS" et "l'envie d'enseigner" les a prioritairement conduites à choisir cette profession. Le métier de "guide-accompagnateur" est évoqué comme étant le plus proche de celui d'enseignant d'EPS. Ainsi, les femmes seraient davantage organisées autour d'une identité "d'enseignante-formatrice", nécessitant une «ouverture au projet pédagogique, à l'établissement et à la diversité des APSA», alors que les hommes semblent davantage centrés sur ce qui relève d'une "compétence sportive" pour enseigner l'EPS.

Au niveau de l'âge, l'étude met en relief la période entre 40 et 50 ans comme la plus favorable aux implications à l'intérieur du système scolaire. De manière générale, les enseignants d'EPS sont fortement engagés dans l'établissement, largement concernés par la dynamique de l'équipe disciplinaire (obligation institutionnelle), et semblent aussi valoriser des modes de relations conviviales au sein du groupe professionnel.

Concernant la pratique sportive, un quart seulement des enseignants d'EPS pratique en compétition et plus de la moitié en loisir. On note aussi que la polyvalence des pratiques l'emporte sur des formes de spécialisation dans une seule APSA. Cette tendance est confirmée pour les pratiques des enfants, plutôt en loisir ou au niveau départemental. Par ailleurs, 20\% des enseignants d'EPS sont entraîneurs en club ; cette caractéristique est corrélée avec un niveau de pratique sportive plus élevé chez leurs enfants (niveau régional et plus). Si la grande hétérogénéïté des cursus de formation initiale et l'évolution disciplinaire amènent les enseignants d'EPS à se former massivement dans le cadre de la formation continue, $20 \%$ le font (aussi) en milieu fédéral, dans une spécialité sportive.

D'un point de vue géographique, on repère des différences notables entre les trois académies choisies. Par exemple, les enseignants de l'académie de Nantes caractérisent davantage la discipline à travers la «centration sur l'élève », la "didactique », "l'évolution des textes officiels » et "les besoins en formation » qu'ils induisent. Les enseignants de l'académie de Créteil évoquent plus particulièrement des changements au niveau des « relations enseignants-élèves » et la nécessité de «s'adapter au contexte social ». Enfin, les enseignants de l'académie de Corse mettent l'accent sur la «variété des APSA » et demandent des formations en ce sens. Critiques vis-à-vis des «conditions de travail», ils choisissent pour eux-mêmes des pratiques sportives polyvalentes et sont plus nombreux à ne pas s'impliquer au sein de l'établissement.

Par ailleurs, la motivation pour le métier reste forte, voire très forte pour les deux tiers de la profession en raison de «l'utilité sociale » de la discipline (enjeu éducatif), de la "richesse des APSA » et de la volonté de «faire réussir» tous les élèves. Lorsque la motivation est moindre, les motifs évoqués sont les «problèmes de santé », les «conditions de travail » et la « relation enseignant-élève ». Dans ce cas l'EPS est aussi perçue comme une discipline en «crise d'identité ».

Enfin, lorsque les enseignants d'EPS expriment des difficultés au quotidien, celles-ci sont "d'ordre pédagogique" (18\%) et "didactique" (17\%); elles touchent aussi aux "conditions de travail" (16\%) et, plus largement, au "malaise de l'école face à la société" (15\%). Les autres items recueillent moins de suffrages : "difficultés relationnelles" avec les enseignants ou l'équipe de direction (10\%), "évolution des textes officiels" (10\%) et "insuffisance de formation" (9\%) soulignent malgré tout des points de tension dans l'exercice du métier. 


\subsection{Représentations professionnelles et valeurs partagées par le groupe professionnel}

Pour les enseignants d'EPS, les changements disciplinaires sont essentiellement liés à la "réflexion sur la didactique de l'EPS" (28\%), à la "centration sur l'élève" (22\%) et à "l'évolution de l'évaluation" (21\%). Les relations enseignant-élèves se sont modifiées et l'EPS, discipline scolaire ne se confond plus avec le sport. En se diversifiant, les pratiques (APSA) constituent des sources de motivation supplémentaires pour les élèves. Enfin, un travail d'équipe leur semble incontournable pour construire le projet pédagogique d'EPS.

Ainsi, la logique institutionnelle, très présente, sert de référence : construite autour d'objets professionnels, elle reste toujours ouverte aux interprétations. C'est donc avec ou contre cette logique que les enseignants d'EPS peuvent se positionner pour défendre leur conception de la discipline.

Par ailleurs, les résultats permettent de réaliser une synthèse des représentations partagées par l'ensemble du groupe professionnel sur le métier. Les deux questions prises en compte pour cette opération offraient un choix de trois items parmi une liste conséquente ; les réponses (calculées sur la base de $100 \%$ ) conduisent à des pourcentages qui peuvent sembler relativement faibles mais qui indiquent de fortes tendances. Dans l'enquête, l'enseignant d'EPS apparaît avant tout comme un "éducateur" (33\%), un "animateur" (16\%) et un "accompagnateur" $(14 \%)^{6}$. Du point de vue des qualités professionnelles (Paquay et Wagner, 1996), il est capable de "construire des contenus pour que l'élève progresse" (22\%) et sait "être en relation" (15\%). D'autres compétences lui sont nécessaires dans l'exercice du métier : il doit "savoir s'adapter" rapidement au contexte du cours (10\%) et bien "connaître la psychologie de l'adolescent" $(10 \%)^{7}$.

Ainsi, une permanence fondatrice de l'identité professionnelle collective traverse toutes les réponses aux questions ouvertes ; elle est organisée autour des valeurs éducatives inscrivant la mission de l'enseignant d'EPS dans une logique de formation, centrée sur une relation enseignant-élève privilégiée dans cette discipline.

\subsection{Identité pour autrui et formes de compromis}

La position identitaire affirmée ci-dessus permet au groupe professionnel d'être reconnu par les différents acteurs de l'école (élèves, enseignants des autres disciplines, équipe de direction, parents, etc.) et intégré au sein de l'école. Il s'agit d'une forme de reconnaissance externe. Les regards croisés sur les enseignants (identité pour autrui) abordés précédemment, indiquent que, pour les élèves, l'EPS sert avant tout à rester en bonne santé, à se défouler, à s'épanouir et à acquérir des techniques sportives pour les loisirs futurs (Hébard, 1986). Il existe donc un écart avec les propos des enseignants d'EPS eux-mêmes qui, pour exister au sein de l'école, revendiquent l'apprentissage de valeurs et s'inscrivent dans la logique institutionnelle mettant l'élève au cœur du dispositif. Une forme de compromis apparaît pourtant. En se positionnant de cette manière, ils rencontrent l'intérêt des parents pour ce qui touche aux valeurs éducatives et à la formation de l'individu (Dorville, 1991 ; Gleyse, 1993). Par ailleurs, en se disant "éducateurs, animateurs et guides", ils intègrent aussi une dimension relationnelle que privilégient les élèves dans cette discipline (Beunard, 1999). Enfin, ils entrent dans une demande d'éducation qui leur est aisément dévolue, y compris par les autres enseignants davantage préoccupés par les savoirs disciplinaires (Dorville, 1991). Cet

\footnotetext{
${ }^{6}$ Les autres résultats se répartissent comme suit : concepteur $(8 \%)$, acteur $(8 \%)$, entraîneur $(6 \%)$, technicien $(6 \%)$, bricoleur $(4 \%)$ militant $(3 \%)$, autre $(2 \%)$.

${ }^{7}$ Les autres réponses se répartissent de la manière suivante : réfléchit sur ses pratiques et analyse leurs effets (8\%), développe des enjeux sociaux dans les situations quotidiennes (7\%), s'engage dans des projets collectifs (7\%), maîtrise bien les APSA (6\%), cherche à évoluer (5\%); les six derniers items choisis obtiennent des scores inférieurs à $3 \%$.
} 
ensemble d'éléments semble constituer une sorte de "label" favorisant l'intégration de l'EPS au sein du système scolaire.

\section{Discussion :}

\subsection{Une continuité disciplinaire fondée sur des valeurs communes mais polysémiques}

Dans les résultats issus de l'enquête par questionnaire, les valeurs éducatives et la relation à l'élève semblent révéler une forme de consensus chez les enseignants d'EPS pour caractériser l'identité disciplinaire. Pourtant, le travail plus en profondeur réalisé avec des entretiens semidirectifs indique les sens multiples donnés à certains termes génériques. Tout d'abord, le terme "d'éducateur" est largement consensuel. Lorsque l'on observe la manière dont les uns ou les autres l'envisagent et le déclinent, des différences significatives apparaissent : "rééduquer des élèves" (Max), "leur apprendre des règles de vie" qui dépassent largement le contexte de l'EPS (Marie) ou "les amener à prendre des responsabilités au sein de l'établissement" (Colette) ne renvoie ni aux mêmes représentations de l'élève, ni aux mêmes objectifs éducatifs.

De la même manière, la relation aux élèves reste un point fortement valorisé par les enseignants, donné le plus souvent comme source de motivation pour ce métier. Cette relation se décline sous différentes formes. Dire collectivement qu'elle est plus forte en EPS n'indique en rien la place réelle donnée à l'élève. Elle peut se faire à travers la simple pratique des APS, conviviale, ludique, dans laquelle l'enseignant associe son rôle à celui d'un animateur sportif, charismatique, porteur de savoir-faire techniques qu'il transmet dans l'action (Pierre, François, Brigitte). Elle peut aussi s'organiser à partir d'une réelle prise en compte de l'élève, à son niveau de ressources. Donner la priorité à la personne se formant et s'intéresser aux dimensions motrices mais aussi socio-affectives et cognitives amène un autre type de relation avec l'enseignant (Paul, Michèle, Claude). Enfin, pour des enseignants comme Marie, la relation aux élèves reste le point d'ancrage, la priorité autour de laquelle s'organisent les autres composantes du métier.

Enfin, les finalités de la discipline constituent une permanence et deviennent ainsi un point d'appui pour l'ensemble des enseignants. Pour justifier de ses choix d'enseignement, chacun interprète les textes à son avantage. En ce sens leur évocation constitue une permanence dans les discours et se fait sous des formes différenciées. En effet, au regard des résultats, l'enseignant peut valoriser l'une ou l'autre des finalités (prioritaire) et supposer ensuite une ouverture aux deux autres (secondaires) en justifiant ses choix pédagogiques. Il peut, par exemple, a) s'attacher à la première finalité de la discipline en privilégiant le développement de la "filière anaérobie" en course de longue durée, en natation, etc. Ceci permettra de construire les bases de l'approche spécifique d'une APSA type demi-fond (deuxième finalité) et servira plus tard à s'entretenir par la pratique de la course à pied, du vélo, de la natation ou du ski de fond (troisième finalité de l'EPS) ; b) valoriser la deuxième finalité de la discipline en disant que l'apprentissage du basket (APSA) permet de développer des qualités d'adresse, d'affiner les prises d'informations, les changements de rythme nécessaires au jeu (première finalité). Cette activité sportive est conditionnée par le respect du règlement. De ce point de vue, le rôle d'arbitre peut aider à assumer des prises de responsabilité (troisième finalité de l'EPS) ; c) valoriser la troisième finalité de la discipline en privilégiant l'apprentissage de la sécurité et en prenant appui sur des APS comme l'escalade ou la gymnastique (deuxième finalité de l'EPS) nécessitant une éducation à cet aspect sécuritaire. En faisant cela, l'enseignant permet de développer des conduites motrices spécifiques intégrant gestion des ressources, coordinations, organisation motrice particulière (première finalité de l'EPS) afin de construire les rôles de pareur (en gymnastique) et d'assureur (en escalade). 
Dans les trois cas, pourtant, il ne s'agit pas du même type d'enseignement. Il est sous-tendu par une conception différente de la discipline et une prise de position vis-à-vis du rapport APSA/EPS. En fait, les programmes, éléments incontournables dans les autres disciplines, restent nécessairement ouverts en EPS (conditions matérielles très particulières d'un établissement à l'autre) ce qui permet à chacun de s'y retrouver, en le justifiant par les textes eux-mêmes.

De plus, la troisième finalité de la discipline apparaît très fortement dans les discours, alors que la préoccupation réelle des enseignants dans les pratiques est faible à cet égard, diversement partagée, prise en compte à l'économie (Cogérino, 1999).

Ces différents constats renvoient à la "logique de l'intégration" (Dubet, 1994) dans laquelle l'acteur interprète la culture comme un ensemble de valeurs assurant une mise en ordre du monde ; pour ce faire, il prend appui sur les principes qui paraissent les plus stables et les moins négociables, maintenant ainsi la continuité de son identité.

Ainsi, ces différents thèmes abordés dans les textes et dans les discours sont largement interprétés par les enseignants et, s'ils constituent une sorte de consensus, ils ne conduisent sans doute pas à des pratiques homogènes.

\subsection{L'élève au cœur des préoccupations éducatives : entre adhésion et résistances, un monde clivé}

En s'intégrant comme discipline d'enseignement au sein du système scolaire, l'EPS s'inscrit dans des changements voulus par l'institution. La place accordée à l'élève a entraîné des conséquences en terme de mise en œuvre de l'enseignement : programmes disciplinaires adaptés, construction à l'échelle de l'établissement d'un projet pédagogique articulant les textes nationaux et le contexte local, réflexion sur les contenus et leurs modes d'évaluation, différenciation pédagogique, etc. L'enquête révèle des prises de positions différenciées sur ces différents objets professionnels, montrant ainsi l'aspect problématique de l'évolution disciplinaire.

En effet, un certain nombre de tensions sont repérables à travers les discours des enseignants : tension entre spécificité et intégration de la discipline au sein du système scolaire, tension entre logique sportive et logique d'apprentissage de l'élève, tension entre pratique et théorie.

Certains enseignants, impliqués au sein du système scolaire, s'engagent dans la réflexion sur l'adaptation des contenus et de l'évaluation au niveau de ressources des élèves, et dans la construction d'un projet pédagogique permettant une plus grande cohérence de l'enseignement. Ils développent une approche polyvalente des APSA pour permettre à l'élève de construire des compétences diversifiées. Le but est alors de faire une éducation "fondamentale" et "équilibrée" en EPS en prenant appui sur des APSA complémentaires, porteuses d'enjeux de formation différents. Ces enseignants se forment régulièrement pour améliorer leur pratique et donner du sens aux apprentissages des élèves.

D'autres, impliqués prioritairement à l'extérieur du système scolaire, se situent en rupture avec l'évolution disciplinaire et opèrent un repli sur la spécificité motrice de la discipline. Certains d'entre-eux, reconnus pour leur compétences sportives, ont suivi une formation initiale relativement courte dans laquelle pratique et pédagogie sportives étaient valorisées. Ces enseignants, souvent plus âgés, sont critiques vis-à-vis de la formation continue actuelle qu'ils estiment trop didactique et s'en désintéressent. L'argumentation s'appuie alors sur l'écart trop important entre théorie et pratique qui rend, de leur point de vue, la formation continue inopérante.

Schéma ${ }^{\circ} 1$

De plus, l'enquête met en relief un monde "clivé" entre tenants des APSA (continuité et spécificité culturelle de l'EPS) et tenants de l'EPS, discipline d'enseignement visant la réussite de tous et cherchant à rendre l'élève acteur de ses apprentissages (changement et intégration). 
Cette centration sur l'élève amène une réflexion didactique en lien avec des procédures d'évaluation formative, de mise en projet de l'élève, de gestion des différences, etc.

Certains enseignants, de par leur histoire et leurs ancrages sont prêts à faire ce pas, d'autres "restent à la porte" et justifient leur position "extérieure" par la construction d'un monde clivé, dans lequel ils ont choisi leur camp. En fait ce clivage est lié à la nécessité de séparer, de disjoindre, pour se situer et créer un "univers à justification simple" (Derouet, 1992) : il y a ceux qui ont raison et ceux qui se trompent. Par exemple, lorsque les enseignants restent dans une logique de l'activité physique, la gestion des groupes mixtes pose problème ; cette difficulté est d'autant plus forte face à l'utilisation d'APSA plus "traditionnelles" et performatives (de type athlétisme, natation ou sports collectifs). La prise en compte du seul résultat montre alors des différences entre garçons et filles, vécues comme insurmontables par l'enseignant. Elle se double souvent d'un oubli de certaines activités moins "classantes" parce que moins "masculines" : activités de pleine nature, activités physiques artistiques ou activités gymniques.

Par ailleurs, si les enseignants signalent de façon massive la réflexion sur la «didactique de $l^{\prime} E P S »$ comme un point d'évolution disciplinaire, cela ne signifie pas pour autant qu'ils y adhèrent. Ce découpage des contenus, leur mise à plat et leur articulation pose problème pour ceux qui voient dans leur enseignement un acte globalisé, tenant avant tout du charisme et ne nécessitant pas forcément l'explicitation des contenus. Le pôle didactique est alors opposé au pôle pédagogique et non pensé comme complémentaire.

Enfin, le projet pédagogique semble envisagé tantôt comme un point d'appui permettant de fédérer les pratiques, de construire une continuité d'enseignement et de donner des repères aux élèves. Dans d'autres cas, il reste un produit purement formel, sans incidences sur les pratiques. Pour les enseignants inscrits dans cette orientation, on repère une centration forte sur les APSA porteuses de valeurs en elles-mêmes, d'où la nécessité de bien les maîtriser pour les enseigner.

Ainsi, lorsqu'il est question "d'accueillir un remplaçant" ${ }^{8}$, les clivages se poursuivent : si enseigner est un art, peu importe les projets et repères pour l'élève. La relation se construit dans «l'ici et maintenant». Si enseigner est une véritable profession, le professionnel explicite ses contenus, les articule à la dynamique de l'établissement et aux caractéristiques des élèves. Il donne des informations au remplaçant pour structurer son action pédagogique et s'assurer d'une relative continuité de l'enseignement.

De la même manière, l'évaluation formatrice est perçue comme une perte de temps par certains enseignants qui valorisent les pratiques pour elles-mêmes et ne voient pas l'utilité pour l'élève de se situer, de réfléchir sur son action. Dans ce cas, les procédures d'observation, de co-évaluation sont considérées comme inefficaces. De plus, elles nécessitent la clarification de critères pour passer de l'implicite à l'explicite. Cette démarche pose sans doute problème à ces enseignants ou reste, pour eux, sans fondement.

\subsection{Entre rapport aux textes et focalisation sur un représentant particulier : l'inspecteur} Pour beaucoup d'enseignants, les textes, relativement vagues et imprécis, sont considérés comme "de grosses balises, laissant une marge de manœuvre importante »(Mathieu) ; ils les interprètent et s'en inspirent plus ou moins dans la réalité de leurs pratiques. Pour d'autres, ils sont lus partiellement ou en surface et, notamment au lycée, ne servent qu'à entrer dans l'exigible (projet pédagogique et modalités d'évaluation explicitées). Pour certains enfin, qui

\footnotetext{
${ }^{8}$ Une question était proposée durant l'entretien : "si vous deviez accueillir un remplaçant, que lui diriez-vous pour l'aider au niveau de sa pratique dans cet établissement?". Cette entrée permettait d'identifier des axes prioritaires pour l'enseignant : centrés plutôt sur la classe, l'APSA, la discipline, l'équipe et le projet EPS, la dynamique de l'établissement, etc.
} 
se situent en résistance avec l'évolution disciplinaire, les textes ne sont pas lus ou très partiellement. En fait, ils ne constituent jamais un point d'appui, situés au niveau des discours et très loin des pratiques.

Dans ce cas, la personne représentante de l'institution (l'IPR) devient l'objet de toutes les focalisations alors que l'on sait son faible poids dans les pratiques quotidiennes. L'inspecteur est perçu comme l'ultime miroir sur lequel l'enseignant lit ses décalages avec les textes. Pour peu que cet inspecteur souligne la mission des enseignants et s'oppose à une éducation physique qui ne serait qu'une juxtaposition d'activités, il met momentanément l'enseignant en difficulté. La situation d'inspection est alors ressentie comme une intrusion dans la vie de l'enseignant, et la grille d'évaluation vécue comme formelle et inopérante. La non prise en compte du contexte est soulignée avec véhémence et l'outil remis en cause de façon catégorique (déni permettant de gérer les dissonances).

\subsection{Une identité professionnelle construite entre intégration et spécificité}

Les textes régissant la discipline semblent bien intégrés car ils sont un levier important pour la profession et sa reconnaissance. L'enseignant de mathématiques ou de français n'a pas besoin de justifier son existence au sein de l'école. L'EPS, pour ne pas se confondre avec les pratiques sportives ou de loisir extérieures à l'école, souvent plus attrayantes pour ceux qui les choisissent, doit insister sur les enjeux éducatifs de la discipline et la prise en compte des potentialités réelles de l'élève.

Mais, dans le même temps, cette position oblige les enseignants à mettre à distance une spécificité motrice qui les a construits. Nous rejoignons donc cette hypothèse selon laquelle le rapport au corps est minoré en EPS dans les discours généraux (Gleyse, 1993), alors qu'il semble fondateur d'une identité partagée et d'une culture commune.

Lorsque la référence au corps, relativement occultée dans les réponses au questionnaire ${ }^{9}$, est abordée à l'occasion des entretiens, les enseignants expriment des positions fort différentes. Les APSA relèvent bien d'une culture commune. Leur pratique au sein des clubs et dans le cursus de formation fédère les enseignants et participe d'un fonds commun partagé (Couturier et Duret, 2000). Mais le statut qui leur est donné dans le cadre de l'enseignement est ensuite très variable d'un enseignant à l'autre. L'enquête permet de repérer des enseignants qui revendiquent une identité sportive valorisante dans la mesure où elle leur a permis de réussir dans des études courtes grâce à leurs compétences sportives (François, Brigitte) ; par voie de conséquence, ils donnent aux APSA un statut particulièrement important. D'autres enseignants placent l'élève au centre des préoccupations, l'APSA ne devenant qu'un moyen de se développer, de progresser avec les autres et vis-à-vis de soi-même. Certains d'entre eux ont eu des lacunes importantes ou un plus faible "capital moteur" (Claude). Enfin, apparaissent des enseignants qui s'inscrivent avant tout dans une approche polyvalente permettant à l'élève de construire des compétences diversifiées dans des APSA porteuses d'enjeux de formation différents. Le but est alors de faire une éducation "fondamentale" et "équilibrée" en EPS (Paul, Marie, Claude, Samuel) en prenant appui sur des APSA complémentaires.

L'association sportive, autre spécificité, est aussi envisagée de différentes manières. Si pour Brigitte (1994), elle est le moyen de reconstruire au sein du lycée une équipe de club, elle permet à Marie et à l'équipe enseignante d'approfondir ce qui se fait en EPS en privilégiant la diversité sur la spécialisation. Quant à Colette, son action au sein de l'Association sportive vise un investissement des élèves dans des tâches diverses permettant de prendre des responsabilités et de construire une « citoyenneté en acte ».

\footnotetext{
${ }^{9}$ Dans l'enquête par questionnaire, la variable 52 permettait de caractériser l'EPS. Ce qui renvoie à la spécificité motrice n'apparaît qu'en troisième et quatrième position : 1) valeurs éducatives : santé, sécurité, citoyenneté, autonomie, citoyenneté $(16 \%)$; 2) logique institutionnelle : programme, projet pédagogique, didactique, évaluation $(12 \%) ; 3)$ développement moteur $(10 \%) ; 4)$ APSA $(7 \%)$.
} 
Enfin, le "mythe du terrain" qui selon Prévost (1991) organise la profession et lui donne le sentiment d'appartenir à une "tribu" nous semble particulièrement opérant pour les enseignants en décalage avec l'évolution disciplinaire ; cela les conduit à des clivages forts entre théorie et pratique, révélant au fil du discours des réseaux de représentations relativement significatifs. D'un côté, les enseignants «en décalage » avec l'évolution disciplinaire valorisent la pratique, le jeu, la technique. Pour eux, tous les élèves aiment le sport. Les APSA doivent être maîtrisées pour être enseignées, au risque d'en restreindre le choix. Le projet pédagogique ne sert à rien : enseigner est un art qui se vit dans l'interaction. De l'autre, les enseignants inscrits dans l'évolution disciplinaire ressentent d'abord les élèves comme différents, ce qui suppose de réfléchir à un traitement didactique des APSA (contenus pour que l'élève progresse) et à des formes d'évaluation adaptées. Le projet pédagogique est envisagé comme une mise en cohérence permettant des continuités, y compris lorsqu'un remplaçant prend le relais de l'enseignant : enseigner est une profession qui suppose conceptions et mises en œuvre, analyses et régulations. Le « terrain » est alors évoqué comme résistant parce que les élèves contournent parfois le projet de l'enseignant et n'entrent pas dans les processus d'apprentissage attendus (Colette, Paul, Michèle).

Cette mise en relief des différences en terme de rapport à la logique institutionnelle n'empêche nullement les enseignants d'EPS d'afficher une cohérence pour ce qui est extérieur au groupe professionnel. Lorsque la discipline est en danger (diminution des horaires, perte de certains acquis comme l'association sportive, etc.) les enseignants d'EPS sont capables de se mobiliser. Pour dépasser les clivages et retrouver une unité, ils se réfèrent aux finalités de la discipline, aux valeurs éducatives et à la relation privilégiée avec les élèves, se situant alors comme des acteurs particuliers et indispensables au sein de l'école.

Ainsi, l'identité professionnelle collective se construit sur un jeu de tensions entre continuité et changement, entre spécificité et intégration de la discipline au sein du système scolaire. Par ailleurs elle se constitue sur des formes de représentations collectives et sur des valeurs partagées, dans le but de défendre le groupe professionnel au sein de l'école et plus largement, vis-à-vis de la société.

\section{Bibliographie}

Abric, J.C. (1976). Jeux, conflits et représentations sociales. Cousset, Del Val.

Abric, J.C. (1994). Pratiques sociales et représentations. Paris, Presses Universitaires de France.

Arnaud, P. (1983). Les savoirs du corps. Lyon, Presses Universitaires de Lyon.

Arnaud, P. (1989). Contribution à une histoire des disciplines d'enseignement. Revue française de pédagogie, 89, 29-34.

Barbier, J.M. \& Galatanu, O. (1998). Action, affects et transformation de soi. Paris, Presses Universitaires de France.

Barbier, J.M. (1996). De l'usage de la notion d'identité en recherche. Education permanente, 128, 11-26.

Berger, G. (1989). Eléments de réflexion pour des dynamiques de changement dans la formation des enseignants : la notion d'identité professionnelle. Recherche et formation, 6, 17-22.

Beunard, B. (1999). Que pensent les lycéens de l'Education Physique ? Revue Education Physique et Sport, 280, 76-79.

Blin, J.F. (1997). Représentations, pratiques et identité professionnelle. Paris, L'harmattan.

Camilleri, C. (Ed.) (1990). Stratégies identitaires. Paris, Presses Universitaires de France.

Cogérino, G. (1999). Apprendre à gérer sa vie physique. Paris, Presses Universitaires de France. 
Couturier, C. \& Duret, P. (2000). Enseigner l'EPS : identité professionnelle à l'épreuve du sport. Paris, Centre National d'Etudes EPS et Société.

Demazière, D. \& Dubar, C. (1997). Analyser les entretiens biographiques. Paris, Nathan.

Derouet, J.L. (1992). Ecole et justice : de l'égalité des chances aux compromis locaux. Paris, Métailié.

Dorvillé, C. (1991). Représentations de l'enseignant d'EPS par les parents d'élèves et les professeurs. STAPS, 24, 7-23.

Dubar, C. (1991). La socialisation : construction des identités sociales et professionnelles. Paris, Armand Colin.

Dubet, F. (1994). Sociologie de l'expérience. Paris, Seuil.

Flament, C. (1987) Pratique et représentations sociales, in J.L. Beauvois., R.V. Joule \& J.M. Monteil (Eds), Perspectives cognitives et conduites sociales, Fribourg, Del Val, 143150 .

Gleyse, J. (1993). Les représentations de l'Education Physique, en France, chez les acteurs du système éducatif, in G. Bui-Xuan. \& J. Gleyse (Eds.). Enseigner l'EPS, ClermontFerrand, Editions AFRAPS, 181-189.

Hébrard, A. (1986). L'éducation physique et sportive. Réflexion et perspective. Paris, Editions Revue EPS / STAPS.

Ion, J. (1990). Le travail social à l'épreuve du territoire. Toulouse, Privat.

Jodelet, D. (1984). Représentations sociales : phénomènes, concept et théorie, in S.

Moscovici, Psychologie sociale, Paris, Presses Universitaires de France, 357-378.

Jodelet, D. (1989). Les représentations sociales. Paris, Presses Universitaires de France.

Josse, A. (1975). Un enseignant comme les autres ? Esprit, 5, 657-671.

Mead, G.H. (1934). Mind, Self and Society. Chicago, University of Chicago Press.

Mias, C. (1998). L'implication professionnelle dans le travail social. Paris, L'Harmattan.

Moliner, P. (1993). L'induction par scénario ambigu. Une méthode pour l'étude des représentations sociales. Revue internationale de psychologie sociale, 6, 7-21.

Moscovici, S. (1961). La psychanalyse, son image et son public. Paris, Presses Universitaires de France.

Prévost, C.M. (1991). L’Education Physique et Sportive en France. Paris, Presses Universitaires de France.

Paquay, L. \& Wagner, M.C. (1996). Compétences professionnelles privilégiées dans les stages et en vidéo-formation, in L. Paquay., M. Altet., E. Charlier \& P. Perrenoud (Eds.). Former des enseignants professionnels, Bruxelles, De Boeck, 154-179.

Roux-Perez, T. (2001). Des processus de construction de l'identité professionnelle des enseignants d'EPS : entre appartenance au groupe, expériences professionnelles singulières et recompositions identitaires à l'échelle du temps. Thèse de doctorat non publiée, Université de Nantes.

Roux-Perez, T. (in press). Processus de construction de l'identité professionnelle des enseignants d'Education Physique et Sportive. Recherche et Formation. Paris, INRP.

Roux-Perez, T (2003). Identité professionnelle et modes d'implication privilégiés chez Les enseignants d'EPS. Revue Les Sciences de l'Education. Pour l'Ere Nouvelle. Vol 36, 4. Caen, CERSE. pp 37-68.

Sainsaulieu, R. (1977). L'identité au travail. Les effets culturels de l'organisation. Paris, Presses de la Fondation nationale des Sciences politiques.

Tap, P. (1980). Identité individuelle et personnalisation, identité collective et changements sociaux. Toulouse, Privat.

Tap, P. (1998). Marquer sa différence, in J.C Ruano-Borbalan, L'identité : l'individu, le groupe, la société, Auxerre, Sciences Humaines, 65-68. 
Schéma $\mathrm{n}^{\circ} 1$ : Système de tensions au sein du groupe professionnel des enseignants d'EPS (Roux-Perez, 2001, 580)

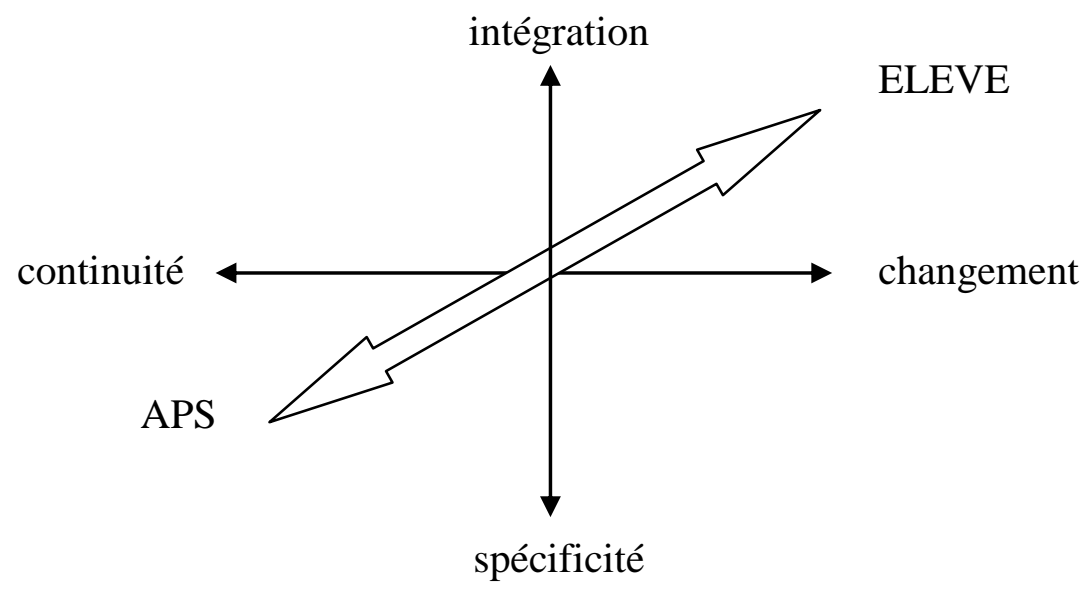

